\title{
The serum proteomics tracking of hepatocellular carcinoma early recurrence following radical resection
}

\author{
Hongzhi Liu ${ }^{1,2, *}$ \\ Hui Chen ${ }^{1, *}$ \\ Xiaomo $\mathrm{Wu}^{3,4}$ \\ Ying Sun ${ }^{2}$ \\ Yingchao Wang ${ }^{2}$ \\ Yongyi Zeng ${ }^{1,2}$ \\ Geng Chen ${ }^{2}$ \\ Xiaolong $\mathrm{Liu}^{2}$ \\ Xiaohua Xing ${ }^{2,5}$ \\ Bixing Zhao ${ }^{2}$ \\ Jingfeng Liu ${ }^{1,2}$
}

'Department of Gastrointestinal Surgery, The First Affiliated Hospital of Fujian

Medical University, Fuzhou, 350007, People's Republic of China; ${ }^{2}$ The United Innovation of Mengchao Hepatobiliary Technology Key Laboratory of Fujian Province, Mengchao Hepatobiliary Hospital of Fujian Medical University, Fuzhou 350025, People's Republic of China; ${ }^{3}$ Department of Biomedicine, University of Basel, Basel 4056, Switzerland; ${ }^{4}$ Dermatology Institute of Fuzhou, Dermatology Hospital of Fuzhou, Fuzhou 350025, People's Republic of China; ${ }^{5}$ The School of Basic Medical Sciences, Fujian Medical University, Fuzhou 350004, People's Republic of China

*These authors contributed equally to this work

Correspondence: Xiaohua Xing; Bixing

Zhao

Mengchao Hepatobiliary Hospital of Fujian Medical University, Xihong Road 3/2,

Fuzhou, Fujian Province, 350025, People's

Republic of China

Tel +86 59l 83705927

Fax +86591 83705927

Email xingxiaohua2006@163.com;

bixingzhao@gmail.com
This article was published in the following Dove Press journal:

Cancer Management and Research

Purpose: There is still lacking of highly sensitive and specific biomarkers for the prediction of hepatocellular carcinoma (HCC) early recurrence, which has hindered further improvement of the clinical outcomes. We aim to find highly sensitive and specific biomarkers for the prediction of HCC recurrence.

Patients and methods: By using isobaric tags for relative and absolute quantitation (iTRAQ)-based multidimensional liquid chromatography-tandem mass spectrometry (2D LC-MS/MS) technique, we have quantitatively investigated and monitored the proteome alterations of a series of serum after radical resection during the follow-up of 4 HCC patients. Results: A total of 27 differentially abundant proteins (DAPs) in serum were identified to be closely associated with the early recurrence of HCC, and these DAPs were particularly concentrated within ERK1/2 and nuclear factor- $\kappa$ beta signaling pathways, suggesting the dysregulation of these two pathways played an important role in the pathological process of HCC early recurrence. Further investigation of a cohort of patients confirmed that the high serum level of PGK1 was closely associated with HCC early recurrence and poor prognosis. In addition, the serum level of PGK1 could be complementary with AFP to further improve the sensitivity and specificity for predicting the relapse of HCC.

Conclusion: PGK1 might be an independent factor for the recurrence of HCC. And the PGK1 could be complementary with AFP to further improve the sensitivity and specificity in prognostic prediction of HCC relapse.

Keywords: hepatocellular carcinoma, early recurrence, serum proteomics, iTRAQ, PGK1

\section{Introduction}

Hepatocellular carcinoma (HCC) is the sixth most common malignancy and the second leading cause of cancer-related death worldwide. ${ }^{1,2}$ Although after decades of efforts, surgical radical resection is still the first choice and the best strategy for HCC therapy. ${ }^{3}$ However, the prognosis of HCC patients after surgical treatment is still unsatisfactory due to high recurrence rate. ${ }^{4,5}$ According to recent studies, the 5 years' recurrence rate of HCC after radical resection is still as high as more than $80 \%$, and the 5 years' overall survival rate of $\mathrm{HCC}$ is still less than $30 \%$. $^{4,6,7}$ Therefore, screening new biomarkers or molecular signatures for predicting and monitoring the early recurrence of HCC after surgical resection are crucial to improve its management and longterm survival.

Serum AFP has been widely used as a biomarker to diagnose and monitor the malignancy of $\mathrm{HCC},{ }^{8-11}$ but its application and interpretation are however constrained 
due to its relatively limited sensitivity and specificity. ${ }^{9,11-13}$ The high-throughput quantitative proteomic technique has emerged as a powerful tool for systematically interrogating most of the proteins or peptides in a biological system. ${ }^{14-16}$ Serum proteomics has become increasingly important in various applications including screening candidate biomarkers for HCC early diagnosis; ${ }^{17-19}$ however, there are few studies focused on screening biomarkers associated with the early recurrence of HCC.

In this study, we performed isobaric tags for relative and absolute quantitation (iTRAQ)-based quantitative proteomics to investigate a series of serum proteomes after radical resection to screen potential biomarkers for predicting HCC early recurrence. In contrast to previous approaches that the sera from different patients are pooled and mixed before subjecting to proteomic analysis, ${ }^{17,18}$ we employed time-course proteomic profiling strategy for each individual patient during the following-up. Our approach could gain a high yield of individual information with temporal resolution on the alterations of serum proteome and thereby improve the specificity and sensitivity of screening potential biomarkers associated with HCC early recurrence.

\section{Patients and methods}

\section{Patients}

All HCC patients enrolled in the serum proteomic analysis and validation experiments received radical HCC surgery at the Mengchao Hepatobiliary Hospital of Fujian Medical University from February 2014 to December 2016. All included patients met the following eligibility criteria: 1) the patient was diagnosed with HCC by postoperative pathological examinations; 2) preoperative serum HBsAg (hepatitis B surface antigen) positive, but $\mathrm{HCV}$ (hepatitis $\mathrm{C}$ virus) negative; 3) the patient had no other therapies before surgery, including TACE, radio frequency ablation and radiotherapy; 4) the patient received standard radical resection: ${ }^{20}$ no distal metastasis was revealed in both pre- and intraoperative examinations; no lesion was found in the rest of the liver during intraoperative ultrasonic scan; no visible cancerembolus in the hepatic portal vein or primary venous branch; no cancer cells were found in the incisal margin at the postoperative pathological examinations, the encapsulation of tumor tissue was intact and the boundary of tumor tissue was distinct; 5) after surgery, the HCC patient was monitored for relapses every 3 months in the first year and every 6 months in the second year by the ultrasonic and CT scan, magnetic resonance imaging and serum AFP test and 6) the patient was followed at least four times before recurrence was found.

\section{Depletion of high-abundant proteins}

Serum samples of three recurrent HCC patients and one nonrecurrent $\mathrm{HCC}$ patient (defined A, B, C and D, respectively) have been collected at the time points of 1 month, 3 months, 6 months and 9 months after radical resection. Details of the major clinical and pathological features of these four HCC patients are shown in Table S1. Before the proteomic analysis, the serum samples were subjected to deplete high-abundant proteins using Human 14 Multiple Affinity Removal System Column (Agilent Technologies, Santa Clara, CA, USA) according to the manufacturer's instructions. The SDS-PAGE and label-free quantitative proteomics were applied to analyze the removal efficiency.

\section{Proteomic analysis}

The serum proteins were digested by sequence-grade modified trypsin (Promega, Madison, WI,USA) and then labeled using chemicals from the iTRAQ reagent kit (ABSCIEX, Framingham, MA, USA) as follows: the four time points of patient A were labeled with 113, 114, 115 and 116 isobaric tags, respectively; the four time points of Patient B were labeled with 117, 118, 119 and 121 isobaric tags, respectively; Patient $\mathrm{C}$ and Patient D were labeled by another iTRAQ kit similarly, as illustrated in Figure 1A. The iTRAQ-labeled peptides were separated using a high $\mathrm{pH}$ reverse-phase chromatography1260 infinity LC system (Agilent Technologies) and analyzed using a EASY-nLC1000 system (Thermo Fisher Scientific, Waltham, MA, USA) connected to a quadrupoleorbitrap mass spectrometer (Q-Exactive Plus) (Thermo Fisher Scientific).

Raw data were processed using Proteome Discoverer (Thermo Fisher Scientific; version 1.4) against a human_database provided by The Universal Protein Resource (http://www.uniprot.org/uniprot, released at 201404-10, with 20,264 entries). The proteins were accepted if peptide FDR is $<1 \%$ and the protein probability is higher than $90 \%$. The differentially abundant proteins (DAPs) in sera were selected by following settings: DAPs were selected based on fold change $>1.2$ or $<0.83$ in both Patient A and Patient $\mathrm{B}$ while exhibiting no such alterations in Patient D.

The Gene Ontology (GO) annotation and the pathway enrichment analysis of DAPs were implemented using the 
A

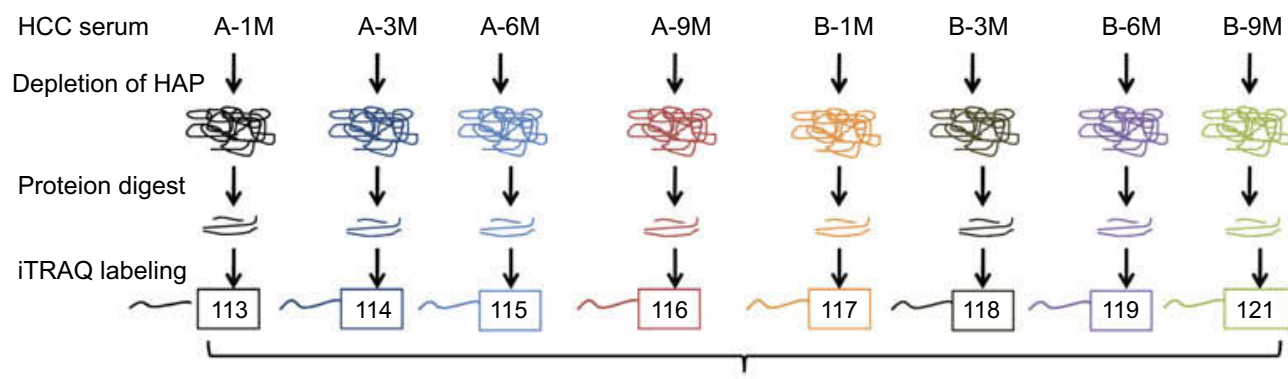

2D LC-MS/MS analysis

Data analysis

Verification
2D LC-MS/MS (Q Exactive Plus)

$\downarrow$ (2 biological repeats for 4 patients)

Bioinformatics analysis

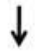

ELISA verification

$\downarrow$

Biomarker of early recurrent $\mathrm{HCC}$

C
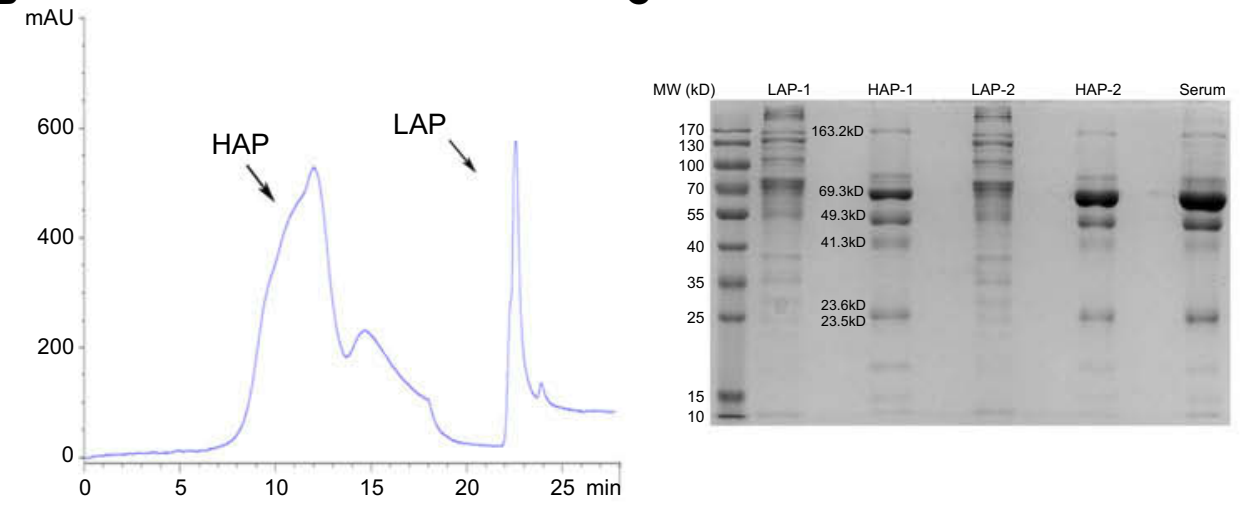

D

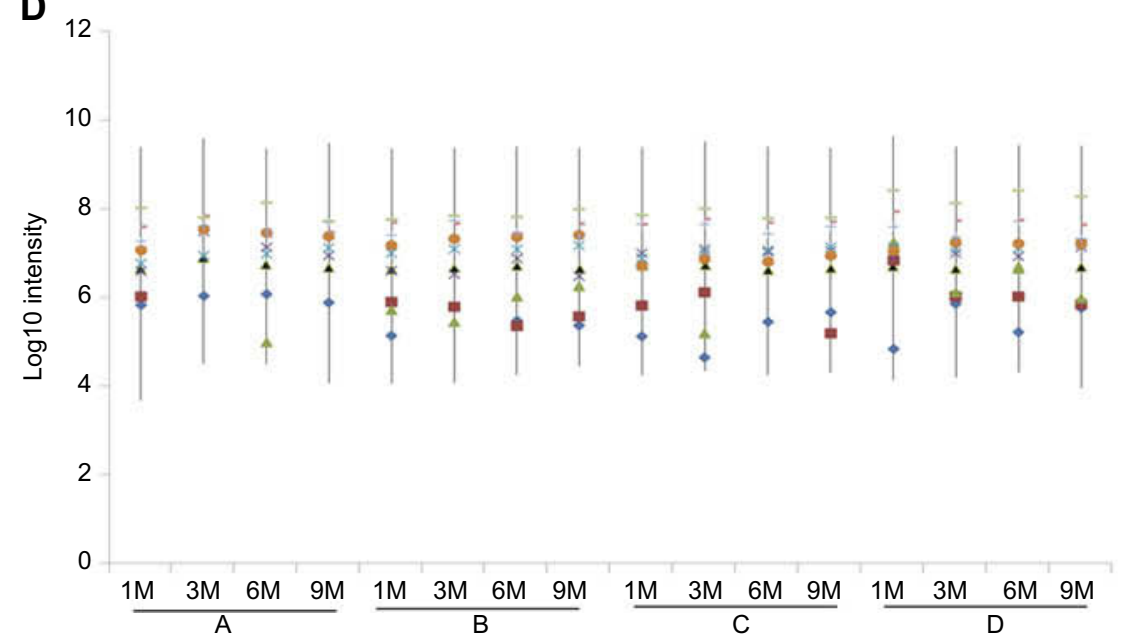

Figure I iTRAQ-based LC-MS/MS proteomic strategy to investigate a series of serum proteomes after radical resection. (A) Schematic view of the experimental design and the iTRAQ plex-labeling. Three recurrent HCC patients and I non-recurrent HCC patient were recruited and the serum samples of these 4 HCC patients have been collected at the timepoints of 1 month, 3 months, 6 months and 9 months after radical resection. Recurrent patients were referred to as Patients A, B and C and nonrecurrent patient as patient D. After depleting HAPs in the serum sample and protein digestion, the resultant peptide mixture was further labeled with chemicals from the iTRAQ kit. Equal amounts of labeled peptide mixture were then analyzed by 2D LC-MS/MS. (B) A chromatogram of MARS-Human I4 Multiple Affinity Removal System Column-based depletion. (C) SDS-PAGE analysis of serum samples before and after removal of HAP by MARS. LAP-I and HAP-I lanes were $5 \mu \mathrm{g}$ of sample loading, LAP-2, HAP-2 and the serum lane was $10 \mu \mathrm{g}$ loading. (D) Label-free analysis of 16 serum samples after the removal of HAPs by MARS. The black triangle is the median of protein concentration and the residual HAPs are marked as colored shapes.

Abbreviations: iTRAQ, isobaric tags for relative and absolute quantitation; LC-MS/MS, multidimensional liquid chromatography-tandem mass spectrometry; HCC, hepatocellular carcinoma; HAPs, high abundance proteins; LAP, low abundance protein. 
online tool DAVID (https://david.ncifcrf.gov/) and Ingenuity Pathway Analysis (IPA) software (version 7.5).

\section{Validation of the PGKI serum level alteration}

The serum levels of PGK1 were examined in a cohort of 82 HCC patients using ELISA assay kit (LifeSpan BioScience Inc., Seattle, WA, USA) according to the manufacturer's protocol. The sensitivity and specificity of the optimal diagnostic cutoff values were obtained according to the gordon index (sensitivity - (1-specific)).

\section{Statistic analysis}

SPSS 19.0 was used for statistical analysis. Multivariate analysis was done using the Cox multivariate proportional hazard regression model with stepwise manner (forward, likelihood ratio). Survival curves were calculated using the Kaplan-Meier method, and the difference was determined using a log-rank test. The $p<0.05$ was considered as statistically significant.

\section{Results}

\section{Depletion of high abundant proteins by MARS}

A typical chromatogram of MARS-based depletion is shown in Figure 1B. The SDS-PAGE result (Figure 1C) indicated that the depletion of high abundance proteins (HAPs) was adequate, as many HAPs basically disappeared in the low abundance proteins (LAPs) lanes compared with the untreated sample. The results of MS-based label-free quantitative proteomic analysis showed that the concentration of residual HAPs was indeed as low as the concentration of LAPs in all 16 serum samples, indicating an effective overcoming of potential mask effects caused by HAPs (Figure 1D).

\section{iTRAQ- based 2D LC-MS/MS analysis of the follow-up serum of HCC patients}

We identified 690 proteins in patient group A/B and 687 proteins in patient group $\mathrm{C} / \mathrm{D}$, with around $80 \%$ of the identified proteins being shared by both groups (shown in Figure 2A). Isoelectric point (IP) analysis of these in total 831 identified proteins demonstrated that acidic proteins were slightly more than that of alkaline proteins (Figure 2B); the molecular weights of the identified serum proteins were relatively small and concentrated between 20 and $60 \mathrm{kDa}$ (Figure 2C).
Furthermore, all identified proteins were processed and analyzed by GO annotation, which demonstrated these identified proteins were mainly distributed in the extracellular region (Figure 2D) and involved in the biological processes that respond to wound, acute inflammatory response and cell adhesion (Figure 2E).

For the analysis of DAPs, the serum proteomes of the first month after surgical resection of all four patients were excluded in the baseline analysis as they exhibited large variations in protein abundance distribution (shown in the Figure 2F), suggesting the unstabilized serum condition of patients shortly after radical resection. We identified 27 HCC recurrence-associated DAPs (as described in the "Materials and methods" section), which were mainly involved in the biological processes that respond to reactive oxygen species stimulus as well as regulating lipid homeostasis (Figure 3A), suggesting the progression of inflammatory and the oxidative stress played an important role in HCC recurrence after radical surgery. ${ }^{21}$ In the clustering analysis of molecular functions, the majority of aberrantly appeared DAPs were involved in the lipase inhibition and the binding with HDL (high-density lipoprotein) particle receptor ${ }^{22,23}$ (Figure 3B), which suggested the abnormalities in lipid metabolism and transportation. Furthermore, the correlation analysis of disease progression revealed these 27 DAPs were mostly linked to metabolic syndrome $\mathrm{X}$ and insulin resistance (Figure 3C), suggesting metabolic disorders were involved in HCC relapse. Additionally, the clustering analysis of regulatory pathways revealed that the aberrant upregulated consumption of glucose was activated through glycolysis pathway (Figure 3D), probably in order to meet the rapid growth requirements of abnormal cells, a phenomenon well known as the Warburg effect in oncology. As shown in Figure $3 \mathrm{E}$ and $\mathrm{F}$, most of these identified DAPs were particularly concentrated within ERK $1 / 2$ and nuclear factor- $\mathrm{kB}(\mathrm{NF}-\mathrm{\kappa B})$ signaling pathways. According to the IPA analysis, the ERK1/2 signaling pathway highly enriched 15 DAPs, including 11 concentration increased and four concentration decreased proteins. Among these, MMPs, PGK1 and TXN are potential biomarkers and involved in the disorder of substance metabolism and energy pathway. Recently, the disorder of substance metabolism and reprogramming of energy metabolism have been found associated with the change of tumor microenvironment, which is thought to be the soil of tumorigenesis, development and recurrence of cancer. And the decreased proteins such as APOA1, APOC2 and APOC3 were involved in lipid 
A

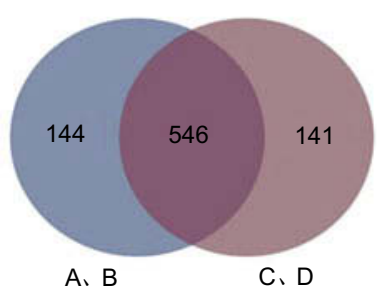

D

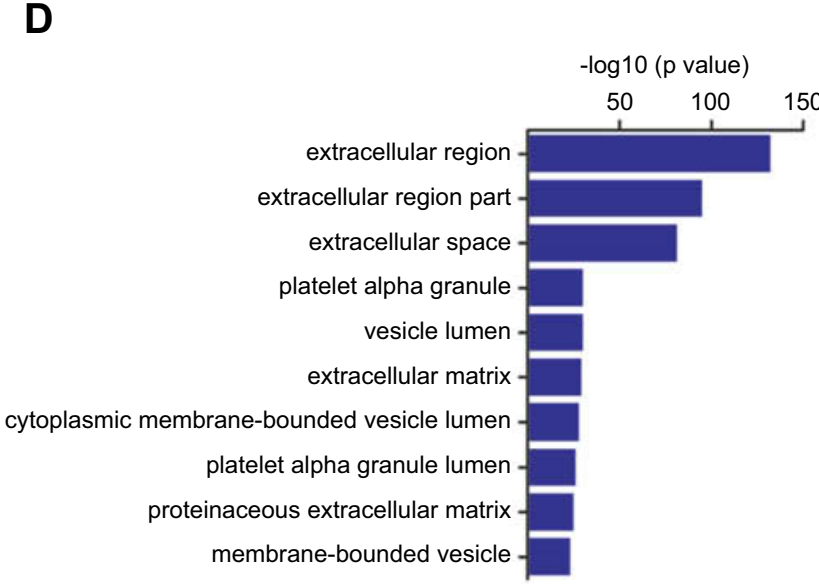

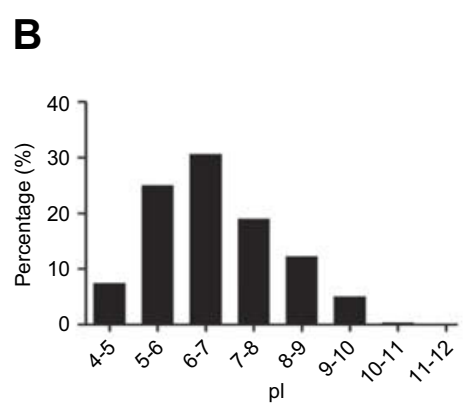

$E$

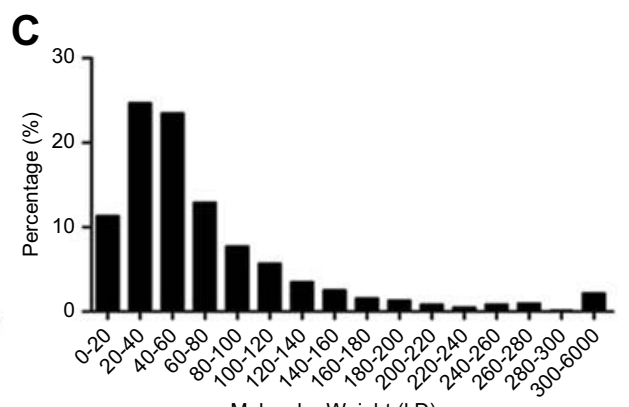

Molecular Weight (kD)

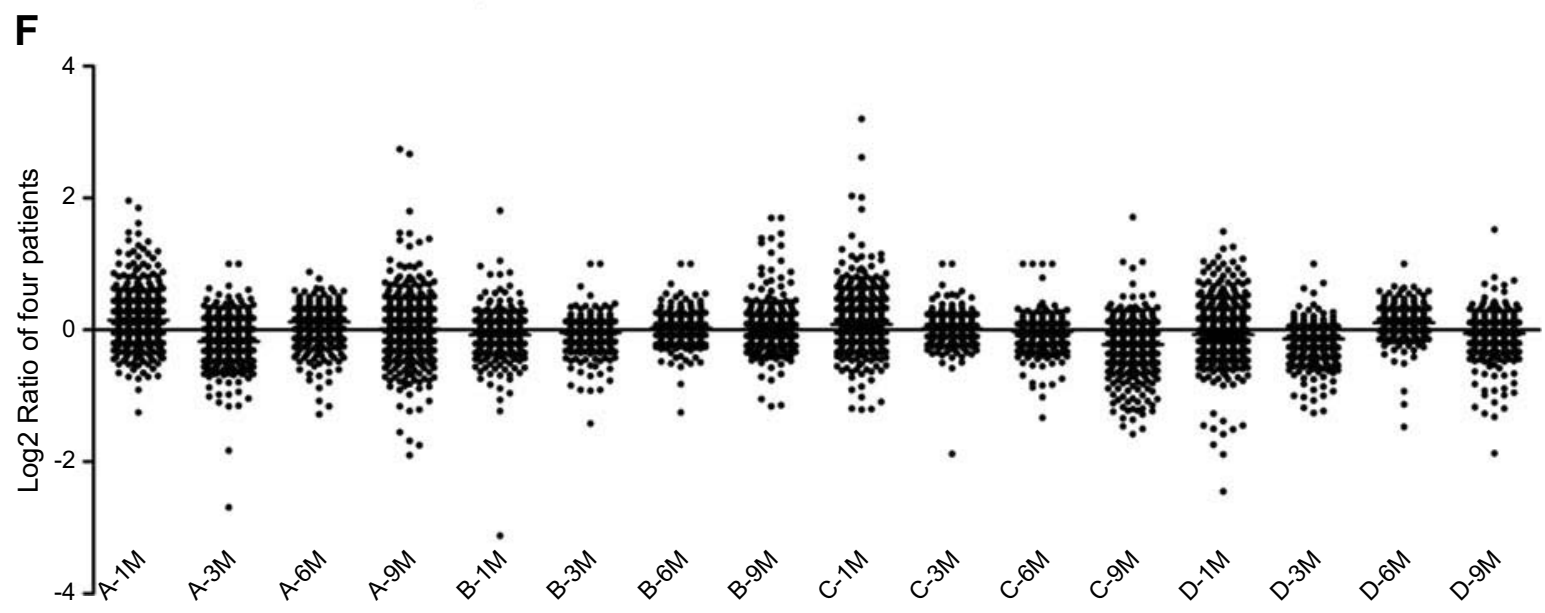

Figure 2 The characteristics of serum proteome. (A) 690 proteins were identified in groups of Patient A/B and 687 proteins in groups of Patient C/D; meanwhile, 546 proteins were identified in both groups. (B) IPs of total 831 serum proteins. IPs were uniformly distributed with the fraction of acidic proteins slightly more abundant than that of alkaline proteins. (C) The molecular weights of $83 \mathrm{I}$ serum proteins. The cell components (D) and the biological processes (E) of all identified proteins were analyzed by GO annotation. (F) The abundances of all proteins identified in 16 serum proteomes.

Abbreviations: IP, isoelectric point; GO, Gene Ontology.

metabolism; SOD1, PGK1, TXN, CAT, CA2 and other increased proteins are mainly involved in redox; in addition, there is MMP9 protein involved in tumor metastasis and other biological processes. All of these results demonstrated that overall biological processes altered in proliferation, differentiation and mobility. Proinflammatory signaling pathway NF$\kappa \mathrm{B}$ also assembled five dysregulated proteins, including BASP1,BPGM, ARHGDIA, IGKC and MAN2A2. Note here, BASP1 that has been recently reported to be a poor prognostic factor for cervical cancer and promote tumor growth was also upregulated in serum level before the recurrence of HCC. Overall, these results suggested the inflammatory response played an extremely important role in $\mathrm{HCC}$ relapse, and the alternations of ERK1/2 and NF- $\mathrm{kB}$ signaling pathways probably synergistically promoted the recurrence and metastasis of $\mathrm{HCC}$ after the curative resection. 
A

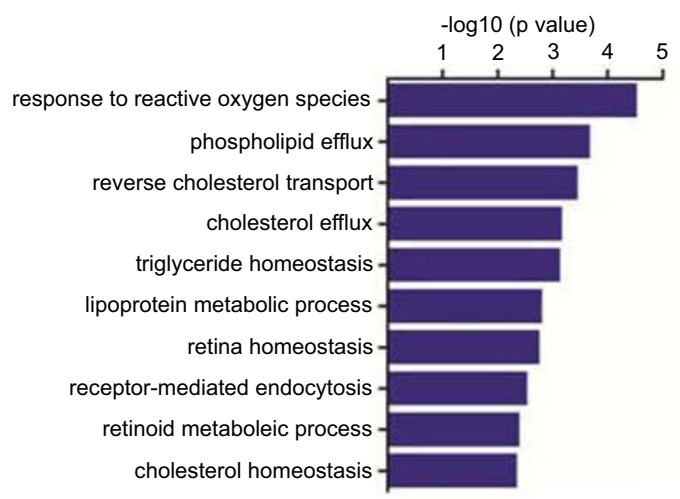

C

E

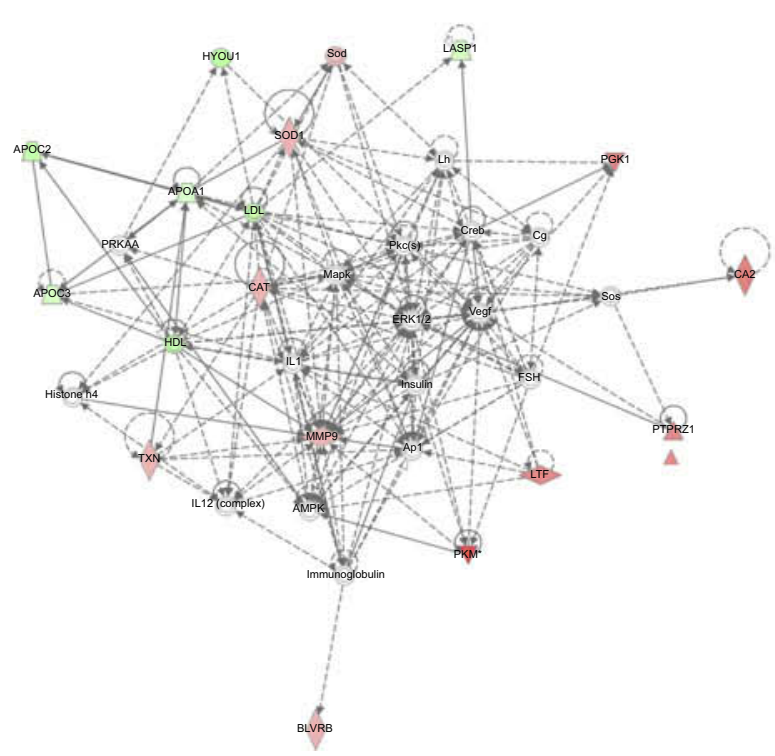

B

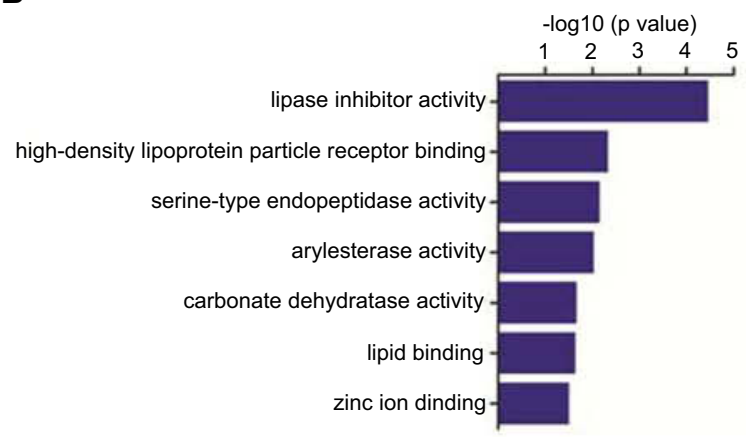

D

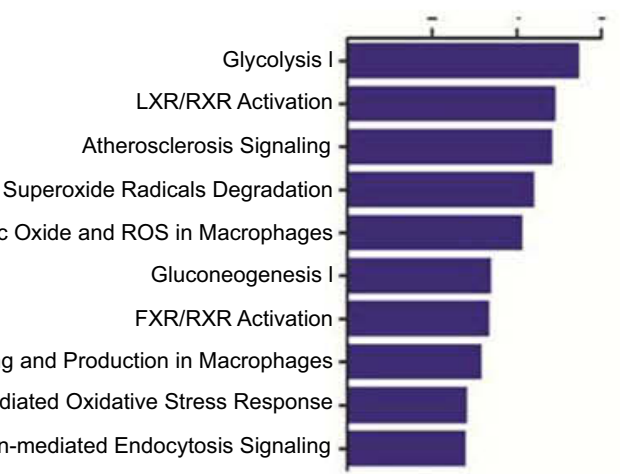

F

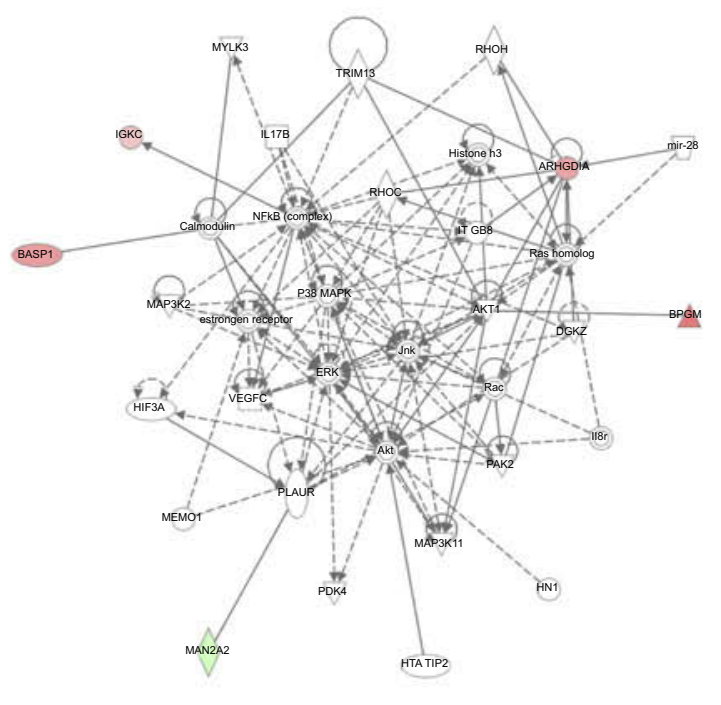

Figure $3 \mathrm{GO}$ and IPA analyses of differentially abundant proteins. The GO annotation of these 27 identified DAPs based on the involved biological processes (A), molecular functions (B), associated disease and disorders (C) and involved molecular pathways (D). IPA of the interaction networks and signaling pathways of these 27 DAPs. The results indicated that the ERKI/2 (E) and NF-KB $(\mathbf{F})$ signaling pathways were involved in the early recurrence of HCC. The red labeling indicates the upregulated proteins and green labeling indicates the downregulated proteins. Solid line shows direct interaction and dotted line shows indirect interaction.

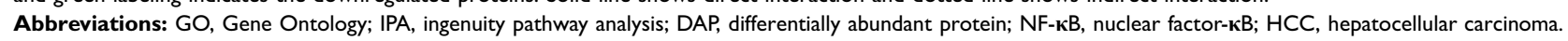




\section{The prognostic value of PGKI in HCC recurrence}

According to the classification based on changing of serum levels, 27 DAPs could be divided into four types: steady increase from 3 to $9 \mathrm{M}$ (Figure 4A), steady increase from 3 to $6 \mathrm{M}$, then sharp increase from 6 to $9 \mathrm{M}$ (Figure 4B), sharp increase from 3 to $6 \mathrm{M}$ but then sharp decrease from 6 to $9 \mathrm{M}$ (Figure 4C), and steady decrease from 3 to $9 \mathrm{M}$ (Figure 4D). As shown in Figure 4B, 13 candidates were suitable for further analysis as prognosis biomarker. Their serum concentration were steadily increased from 3 to $9 \mathrm{M}$, and six of these proteins including PGK1 with the largest fold change were emphatically analyzed. Among these, PGK1 was the unique protein particularly enriched in the ERK $1 / 2$ signaling pathway (Figure 3E), which demonstrated that altered serum PGK1 might play an essential role in the aggressive behavior of HCC by enhancing tumor cell proliferation, anti-apoptosis and migration. PGK1 transfers a phosphate group from 1,3-biphosphoglycerate to ADP to form ATP and 3-phosphoglycerate in the glycolytic pathway, ${ }^{24}$ and it has been previously reported to be involved in tumorigenesis in various cancers through enhancing cellular metabolism. ${ }^{25,26}$ The validation of PGK1 alternation pattern was further performed through blinded analysis in a cohort of 82 HCC patients. Details of the major clinical and pathological features of these patients are shown in Table S2. The univariate analysis indicated that the tumor size, microvascular invasion of tumor, TNM stage, BCLC stage, serum level of PGK1, and serum AFP level were significantly associated with the early relapse of HCC after curative resection; whereas multivariate analysis demonstrated that among all the univariate identified factors only the serum level of PGK1 or AFP was an independent risk factor for the recurrence of HCC (Table 1).

The Kaplan-Meier survival analysis revealed that the serum level of PGK1 could nicely discriminate the prognosis performance of HCC patients, and the lower level of serum PGK1 indicated better prognosis (longer recurrence-free survival, RFS) with the optimal cutoff value of $0.7 \mathrm{ng} / \mathrm{mL}$. Furthermore, the combination of PGK1with AFP could even provide better discrimination and higher performance for the prediction of HCC prognosis (Figure 4E), as the patients with high serum levels of both PGK1 and AFP had significantly shorter RFS compared with the patients who were low with both or only had high serum level of one either PGK1 or AFP alone $(p<0.01)$. Thus, the PGK1 could be complementary with AFP to better predict the prognosis of HCC patients.

Taken together, these results demonstrated that high serum level of PGK1 was closely associated with poor prognosis and recurrence of $\mathrm{HCC}$ after radical resection, and the combination of PGK1 and AFP could significantly improve the predictive capacity of HCC relapse.
A

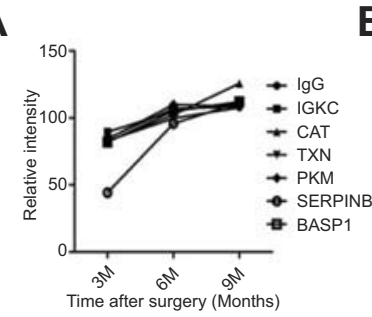

E

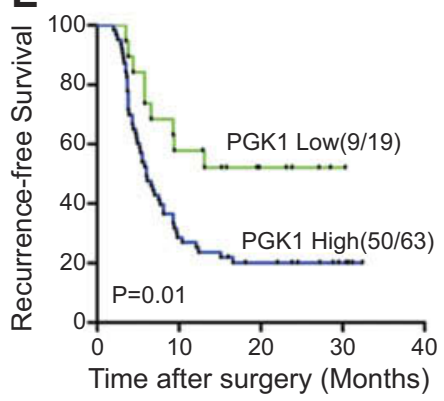

B

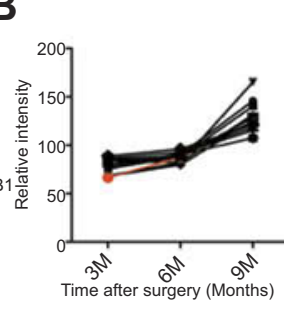

C

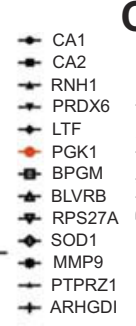

C D

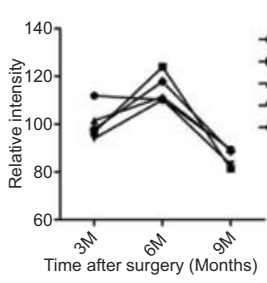

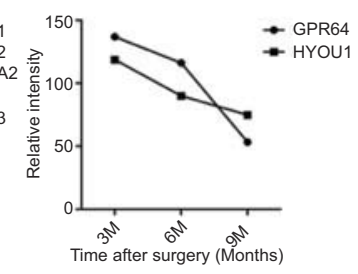
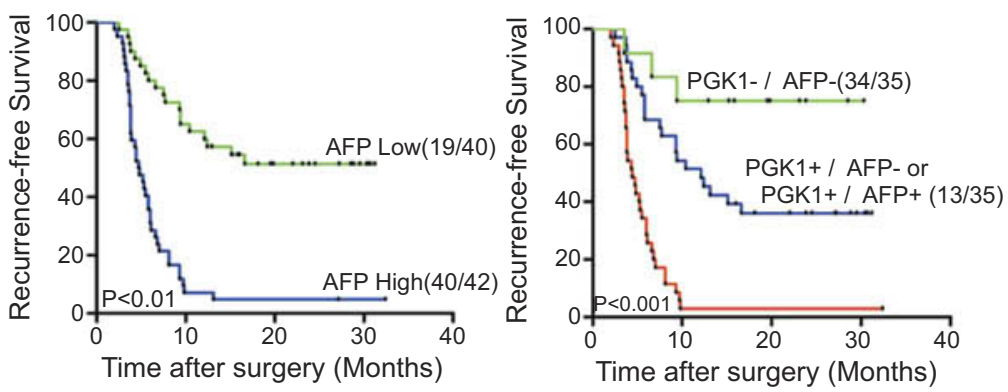

Figure 4 The prognostic value of PGKI in HCC recurrence. Twenty-seven DAPs could be divided into four clusters: steady increase from 3 to 9 M (A), steady increase from 3 to $6 M$ then sharp increase from 6 to $9 M(B)$, sharp increase from 3 to $6 M$ but then sharp decrease from 6 to $9 M(C)$, and steady decrease from 3 to $9 M$ (D). The fold change curve of PGKI was colored in red in the second cluster (B). (E) The prognostic values of PGKI/AFP serum levels and the combination of PGKI/AFP in RFS (logrank test).

Abbreviations: HCC, hepatocellular carcinoma; DAP, differentially abundant protein; AFP, alpha-fetoprotein; RFS, recurrence-free survival. 
Table I Univariate and multivariate logistic regression analysis for risk factors of HCC recurrence

\begin{tabular}{|c|c|c|c|c|c|c|}
\hline \multirow[t]{2}{*}{ Parameter } & \multicolumn{3}{|c|}{ Univariate analysis } & \multicolumn{3}{|c|}{ Multivariate analysis } \\
\hline & OR & $95 \% \mathrm{Cl}$ & $p$ & OR & $95 \% \mathrm{Cl}$ & $p$ \\
\hline Gender & 0.841 & $0.157-4.506$ & 0.840 & - & - & - \\
\hline Age & 1.249 & $0.409-3.812$ & 0.696 & - & - & - \\
\hline Number & 2.256 & $0.674-7.556$ & 0.187 & - & - & - \\
\hline Size & 4.765 & $|.635-| 3.89 \mid$ & $0.004 * *$ & 13.630 & $1.004-185.083$ & 0.050 \\
\hline Gross vascular invasion & 11.282 & $1.416-89.878$ & $0.022^{*}$ & 0.014 & $0-2.654$ & 0.111 \\
\hline Microvascular invasion & 3.033 & $1.121-8.212$ & $0.029 *$ & 2.056 & $0.195-21.629$ & 0.548 \\
\hline Cirrhosis & 1.620 & $0.354-7.41 I$ & 0.534 & - & - & - \\
\hline Differentiation & 1.728 & $0.626-4.768$ & 0.291 & - & - & - \\
\hline AJCC stage & 17.333 & $2.19-137.209$ & $0.007 * *$ & 1.904 & $0.014-264.541$ & 0.798 \\
\hline BCLC stage & 13.327 & $2.86 I-62.088$ & $0.00 I^{* *}$ & 37.874 & $0.811-1768.191$ & 0.064 \\
\hline HBV copies & 0.835 & $0.306-2.281$ & 0.725 & - & - & - \\
\hline PGKI & 4.274 & $1.44-12.683$ & $0.009 *$ & 145.028 & $3.44 I-6 I I 2.214$ & $0.009 * *$ \\
\hline AFP & 22.105 & $4.693-104.127$ & $<0.001^{* * *}$ & 24.097 & $2.40 \mathrm{I}-24 \mathrm{I} .839$ & $0.007^{* *}$ \\
\hline
\end{tabular}

Notes: Multivariate analysis was done using the Cox multivariate proportional hazard regression model with stepwise manner (forward, likelihood ratio). ${ }^{*}<<0.05$; $* *<<0.0 \mathrm{I}$ $* * * p<0.001$.

Abbreviations: AJCC, American Joint Committee on Cancer; BCLC, Barcelona Clinic Liver Cancer; HBV, hepatitis B virus ; AFP, $\alpha$-fetoprotein; -, not available.

\section{Discussion}

High postoperative recurrence rates are the major cause of poor prognosis of $\mathrm{HCC}$ patients after radical resection. ${ }^{27-29}$ Previously, there were some efforts to screen clinicopathological risk factors associated with the recurrence of $\mathrm{HCC}^{30,31}$ However, the clinical outcomes of HCC patients with similar clinical manifestations, similar clinical staging and receiving the same treatments still may be very different after curative resection. ${ }^{6,32}$ Therefore, elucidating the molecular mechanisms underlying HCC recurrence and screening new and reliable molecular biomarkers for discriminating the patients with high risk of relapse are crucial for the improvement of longterm survival of HCC patients after the surgical treatment.

In an attempt to obtain a high yield of individual information with the temporal resolution, we applied time-course profiling based on proteomic techniques for each patient during the follow-up after radical resection. Comparing with the most common proteomic strategy where sera from different patients were pooled and mixed before analysis, ${ }^{17,18}$ we have proven in the concept that our approach allowed to detect very subtle alterations within each patient but could avoid the interference originated from individual differences at some extent. Moreover, the time window to detect specific alterations related with HCC recurrence appeared rather early in this study, which was demonstrated by the comparison between Patient $\mathrm{C}$ and Patient A and Patient B. This observation further supported the advantage of conducting time-course interrogation within each patient.

By monitoring three recurrent patients and one nonrecurrent patient up to 2 years of follow-up based on quantitative iTRAQ proteomics, we found that the abnormalities in lipid homeostasis, upregulated cellular metabolism and stimulated inflammatory reactions all contributed to the pathological process prior to the relapse of HCC. The DAPs might serve as molecular signatures of the recurrence of $\mathrm{HCC}$ after radical resection, but in-depth studies are required for better understanding the underlying molecular mechanisms.

Furthermore, we confirmed the serum levels of PGK1 could be used as a novel prognostic biomarker for predicting the recurrence of HCC after surgical therapy. Elevated levels of PGK1 have been detected in the serum of patients with various malignancies, such as pancreatic cancer, ${ }^{33}$ breast cancer, ${ }^{34}$ gastric cancer ${ }^{35}$ and usually associated with poor clinical outcomes. In our study, high serum levels of PGK1 were proved to be an independent factor for the recurrence of HCC. Additionally, the PGK1 could be complementary with AFP to further improve the sensitivity and specificity in the prognostic prediction of HCC relapse. Taken together, we have revealed the molecular signatures prior to HCC recurrence and provided in-depth information that may help to have a better understanding of this pathological process and to improve the HCC management for long-term survival. 


\section{Conclusion}

By using iTRAQ-based 2D LC-MS/MS technique, we have quantitatively investigated and monitored the proteome alterations of a series of serum after radical resection during the follow-up of four HCC patients. Further investigation of a cohort of patients confirmed that the high serum level of PGK1 was closely associated with HCC early recurrence and poor prognosis. In addition, the serum level of PGK1 could be complementary with AFP to further improve the sensitivity and specificity for predicting the relapse of HCC. We have revealed the molecular signatures prior to $\mathrm{HCC}$ recurrence and provided fundamental information for further in-depth study.

\section{Acknowledgments}

This work is supported by the National Natural Science Foundation of China (Grant no. 81602102, Grant no. 81672376, and Grant no. 81702910); the National Natural Science Foundation of Fujian Province (Grant no. 2017J01159 and Grant no. 2016J01417); the Educational Commission of Fujian Province (Grant no. 2018B013); the Special Projects of Fujian Provincial Finance Department (Grant no. [2014]1262); the Medical Innovation Project of Fujian Province (2016-CX-48), the Scientific Foundation of Fujian Health and Family Planning Department (Grant no. 2016-1-86); the Scientific Foundation of Fuzhou Health Department (Grant no. 2015-S-wq12, Grant no. 2016S-124-7 and Grant no. 2015-S-143-19), and the Fuzhou Health and Family Planning Science and Technology Project (2017-S-wt2).

\section{Disclosure}

The authors report no conflicts of interest in this work.

\section{References}

1. Ferlay J, Soerjomataram I, Dikshit R, et al. Cancer incidence and mortality worldwide: sources, methods and major patterns in GLOBOCAN 2012. Int J Cancer. 2015;136(5):E359-E386. doi:10.1002/ijc.29210

2. Siegel RL, Miller KD, Jemal A. Cancer statistics, 2018. CA Cancer J Clin. 2018;68:7-30. doi:10.3322/caac.21442

3. Jarnagin W, Chapman WC, Curley S, et al. Surgical treatment of hepatocellular carcinoma: expert consensus statement. HPB. 2010;12:302-310. doi:10.1111/j.1477-2574.2010.00182.x

4. Tabrizian P, Jibara G, Shrager B, Schwartz M, Roayaie S. Recurrence of hepatocellular cancer after resection patterns, treatments, and prognosis. Ann Surg. 2015;261:947-955. doi:10.1097/SLA.0000000000000710

5. Shirabe K, Wakiyama S, Gion T, et al. Clinicopathological risk factors linked to recurrence pattern after curative hepatic resection for hepatocellular carcinoma - results of 152 resected cases. HepatoGastroenterology. 2007;54:2084-2087.

6. Wakayama K, Kamiyama T, Yokoo H, et al. Huge hepatocellular carcinoma greater than $10 \mathrm{~cm}$ in diameter worsens prognosis by causing distant recurrence after curative resection. J Surg Oncol. 2017;115:324-329. doi:10.1002/jso.24501
7. Li C, Wen TF, Yan LN, et al. Liver resection versus liver resection plus TACE for patients with hepatocellular carcinoma beyond Milan criteria. J Surg Res. 2017;209:8-16. doi:10.1016/j.jss.2016.09.054

8. Bai DS, Zhang C, Chen P, Jin SJ, Jiang GQ. The prognostic correlation of AFP level at diagnosis with pathological grade, progression, and survival of patients with hepatocellular carcinoma. Sci Rep. 2017;7. doi:10.1038/s41598-017-12834-1

9. Badshah MB, Ghabril M, Fadel W, et al. The role of AFP in screening and diagnosis of viral and non-viral hepatocellular carcinoma (HCC). Hepatology. 2015;62:1136A-1137A.

10. Wu W, Yao DF, Qiu LW, Wu XH. Clinical values of combined hepatoma-specific AFP fraction and AFP-mRNA determination in diagnosis and differentiation of hepatocellular carcinoma. Prog PostGenome Technology; 2006; Hangzhou, China. 443-446.

11. Kelleher PC, Smith CJP, Ajdukiewicz A. Human alpha-fetoprotein (AFP) - lectin-binding heterogeneity and the early diagnosis of primary hepatocellular-carcinoma (PHC). Proceedings of the American Association for Cancer Research; 1983; Philadelphia, PA. 24:240.

12. Sang W, Zhang W, Cui WL, Li XX, Abulajiang G, Li QX. Arginase-1 is a more sensitive marker than HepPar-1 and AFP in differential diagnosis of hepatocellular carcinoma from nonhepatocellular carcinoma. Tumor Biol. 2015;36:3881-3886. doi:10.1007/s13277-014-3030-6

13. Ghouri YA, Mian I, Rowe JH. Review of hepatocellular carcinoma: epidemiology, etiology, and carcinogenesis. J Carcinog. 2017;16:1. doi:10.4103/jcar.JCar_9_16

14. Schubert OT, Rost HL, Collins BC, Rosenberger G, Aebersold R. Quantitative proteomics: challenges and opportunities in basic and applied research. Nat Protoc. 2017;12:1289-1294. doi:10.1038/ nprot. 2017.040

15. Werner T, Becher I, Sweetman G, Doce C, Savitski MM, Bantscheff M. High-resolution enabled TMT 8-plexing. Anal Chem. 2012;84:7188-7194. doi:10.1021/ac301553x

16. Wang YC, Liu HZ, Liang D, et al. Reveal the molecular signatures of hepatocellular carcinoma with different sizes by iTRAQ based quantitative proteomics. J Proteomics. 2017;150:230-241. doi:10.1016/j. jprot.2016.09.008

17. Bhosale SD, Moulder R, Kouvonen P, Lahesmaa R, Goodlett DR. Mass spectrometry-based serum proteomics for biomarker discovery and validation. Methods Mol Biol. 2017;1619:451-466. doi:10.1007/9781-4939-7057-5 31

18. Tsai TH, Song EW, Zhu R, et al. LC-MS/MS-based serum proteomics for identification of candidate biomarkers for hepatocellular carcinoma. Proteomics. 2015;15:2369-2381. doi:10.1002/pmic.201400364

19. Qin X, Chen QP, Sun C, et al. High-throughput screening of tumor metastatic-related differential glycoprotein in hepatocellular carcinoma by iTRAQ combines lectin-related techniques. Med Oncol. 2013;30. doi:10.1007/s12032-012-0420-8

20. Iizuka N, Oka M, Yamada-Okabe H, et al. Oligonucleotide microarray for prediction of early intrahepatic recurrence of hepatocellular carcinoma after curative resection. Lancet. 2003;361:923-929. doi:10.1016/S0140-6736(03)12775-4

21. Mittal M, Siddiqui MR, Tran K, Reddy SP, Malik AB. Reactive oxygen species in inflammation and tissue injury. Antioxid Redox Signal. 2014;20:1126-1167. doi:10.1089/ars.2012.5149

22. Fidge NH. High density lipoprotein receptors, binding proteins, and ligands. J Lipid Res. 1999;40:187-201.

23. Silver DL, Wang N, Xiao X, Tall AR. High density lipoprotein (HDL) particle uptake mediated by scavenger receptor class B type 1 results in selective sorting of HDL cholesterol from protein and polarized cholesterol secretion. J Biol Chem. 2001;276:25287-25293. doi:10.1074/jbc.M101726200

24. Li XJ, Jiang YH, Meisenhelder J, et al. Mitochondria-translocated PGK1 functions as a protein kinase to coordinate glycolysis and the TCA cycle in tumorigenesis. Mol Cell. 2016;61:705-719. doi:10.1016/j. molcel.2016.02.009 
25. Xie HJ, Tong GH, Zhang YP, Liang S, Tang KR, Yang QH. PGK1 drives hepatocellular carcinoma metastasis by enhancing metabolic process. Int J Mol Sci. 2017;18. doi:10.3390/ijms18081630

26. Ai JZ, Huang HZ, Lv XY, et al. FLNA and PGK1 are two potential markers for progression in hepatocellular carcinoma. Cell Physiol Biochem. 2011;27:207-216. doi:10.1159/000327946

27. Zheng J, Chou JF, Gonen M, et al. Prediction of hepatocellular carcinoma recurrence beyond milan criteria after resection. Ann Surg. 2017;266:693-701.

28. Braillon A. Recurrence in early-stage hepatocellular carcinoma: $\mathrm{CpG}$ methylation and smoking. J Clin Oncol. 2017;35:2097-+. doi:10.1200/JCO.2017.72.3932

29. Barrett M, Nathan H, Vankayala H, Bieliauskas SL, Viglianti BL, Frankel TL. Recurrence of hepatocellular carcinoma at surgical incision site: case series and review of literature. Ann $R$ Coll Surg Engl. 2017;99:E177-E179. doi:10.1308/rcsann.2017.0097

30. Singal AG, El-Serag HB. Hepatocellular carcinoma from epidemiology to prevention: translating knowledge into practice. Clin Gastroenterol Hepatol. 2015;13:2140-2151. doi:10.1016/j.cgh.2015.08.014
31. Goh GB, Chang PE, Tan CK. Changing epidemiology of hepatocellular carcinoma in Asia. Best Pract Res Clin Gastroenterol. 2015;29:919-928. doi:10.1016/j.bpg.2015.09.007

32. Lazzara C, Navarra G, Lazzara S, et al. Does the margin width influence recurrence rate in liver surgery for hepatocellular carcinoma smaller than $5 \mathrm{~cm}$ ? Eur Rev Med Pharmacol Sci. 2017;21:523-529.

33. Hwang TL, Liang Y, Chien KY, Yu JS. Overexpression and elevated serum levels of phosphoglycerate kinase 1 in pancreatic ductal adenocarcinoma. Proteomics. 2006;6:2259-2272. doi:10.1002/pmic. 200500345

34. Sun S, Liang X, Zhang X, et al. Phosphoglycerate kinase-1 is a predictor of poor survival and a novel prognostic biomarker of chemoresistance to paclitaxel treatment in breast cancer. $\mathrm{Br}$ $J$ Cancer. 2015;112:1332-1339. doi:10.1038/bjc.2015.114

35. Zieker D, Konigsrainer I, Traub F, et al. PGK1 a potential marker for peritoneal dissemination in gastric cancer. Cell Physiol Biochem. 2008;21:429-436. doi:10.1159/000129635 


\section{Supplementary materials}

Table SI The clinicopathologic characteristics of 4 patients with HCC

\begin{tabular}{|c|c|c|c|c|}
\hline \multirow[t]{2}{*}{ Characteristics } & \multicolumn{3}{|l|}{ Recurrent } & \multirow{2}{*}{$\begin{array}{l}\text { Nonrecurrenent } \\
\text { Patient D }\end{array}$} \\
\hline & Patient A & Patient B & Patient C & \\
\hline Gender & Female & Male & Male & Male \\
\hline Age (years) & 50 & 43 & 51 & 42 \\
\hline Size $(\mathrm{cm})$ & 2 & 3.5 & 4.7 & 3.5 \\
\hline Number & I & I & 1 & I \\
\hline Capsule & Yes & Yes & No & Yes \\
\hline Gross vascular invasion & No & No & No & No \\
\hline Microvascular invasion & No & Yes & No & No \\
\hline Hepatitis B & Positive & Positive & Positive & Positive \\
\hline Cirrhosis & Yes & Yes & Yes & Yes \\
\hline $\operatorname{AFP}(\mathrm{ng} / \mathrm{mL})$ & 3.66 & $1,218.28$ & 2.93 & 2.04 \\
\hline Differentiation & $\mathrm{I}-\mathrm{II}$ & II & III & II \\
\hline AJCC stage & 1 & II & I & I \\
\hline $\mathrm{BCLC}$ stage & $A$ & A & $A$ & A \\
\hline Recurrent time (months) & 12 & 12 & 17 & l \\
\hline
\end{tabular}

Abbreviations: AJCC, American Joint Committee on Cancer; BCLC, Barcelona Clinic Liver Cancer; AFP, $\alpha$-fetoprotein; /, not available; HCC, hepatocellular carcinoma.

Table S2 The clinicopathologic characteristics of 82 patients with HCC

\begin{tabular}{|c|c|c|}
\hline Clinicopathological characteristics & Classify & Cases \\
\hline \multirow[t]{2}{*}{ Gender } & Male & 74 \\
\hline & Female & 8 \\
\hline \multirow[t]{2}{*}{ Age (years) } & $<45$ & 19 \\
\hline & $\geq 45$ & 63 \\
\hline \multirow[t]{2}{*}{ Tumor number } & 1 & 59 \\
\hline & $\geq 2$ & 23 \\
\hline \multirow[t]{2}{*}{ Tumor size $(\mathrm{cm})$} & $<5$ & 39 \\
\hline & $\geq 5$ & 43 \\
\hline \multirow[t]{2}{*}{ Gross vascular invasion } & No & 62 \\
\hline & Yes & 21 \\
\hline \multirow[t]{2}{*}{ Micro-vascular invasion } & No & 34 \\
\hline & Yes & 48 \\
\hline \multirow[t]{2}{*}{ Cirrhosis } & Negative & 8 \\
\hline & Positive & 74 \\
\hline \multirow[t]{2}{*}{ Differentiation } & I-II & 25 \\
\hline & III-IV & 57 \\
\hline \multirow[t]{2}{*}{ AJCC stage } & $I-I I$ & 55 \\
\hline & III-IV & 27 \\
\hline \multirow[t]{2}{*}{ BCLC stage } & A & 47 \\
\hline & $B C$ & 35 \\
\hline \multirow[t]{2}{*}{ HBV copies } & $\leq 1000$ & 36 \\
\hline & $>1000$ & 46 \\
\hline \multirow[t]{2}{*}{$\operatorname{AFP}(\mathrm{ng} / \mathrm{mL})$} & $<400$ & 53 \\
\hline & $\geq 400$ & 29 \\
\hline \multirow[t]{2}{*}{ Recurrence } & Negative & 23 \\
\hline & Positive & 59 \\
\hline
\end{tabular}

Abbreviations: AJCC, American Joint Committee on Cancer; BCLC, Barcelona Clinic Liver Cancer; HBV, Hepatitis B virus; AFP, $\alpha$-fetoprotein; HCC, hepatocellular carcinoma. 


\section{Publish your work in this journal}

Cancer Management and Research is an international, peer-reviewed open access journal focusing on cancer research and the optimal use of preventative and integrated treatment interventions to achieve improved outcomes, enhanced survival and quality of life for the cancer patient.
The manuscript management system is completely online and includes a very quick and fair peer-review system, which is all easy to use. Visit http://www.dovepress.com/testimonials.php to read real quotes from published authors.

Submit your manuscript here: https://www.dovepress.com/cancer-management-and-research-journal 